\title{
Clinical use of whole genome sequencing for Mycobacterium tuberculosis
}

\author{
Adam A. Witney ${ }^{1 *}$, Catherine A. Cosgrove ${ }^{1,2}$, Amber Arnold ${ }^{2}$, Jason Hinds ${ }^{1}$, Neil G. Stoker ${ }^{1}$ and Philip D. Butcher ${ }^{1}$
}

\begin{abstract}
Drug-resistant tuberculosis (TB) remains a major challenge to global health and to healthcare in the UK. In 2014, a total of 6,520 cases of TB were recorded in England, of which $1.4 \%$ were multidrug-resistant TB (MDR-TB). Extensively drug-resistant TB (XDR-TB) occurs at a much lower rate, but the impact on the patient and hospital is severe. Current diagnostic methods such as drug susceptibility testing and targeted molecular tests are slow to return or examine only a limited number of target regions, respectively. Faster, more comprehensive diagnostics will enable earlier use of the most appropriate drug regimen, thus improving patient outcomes and reducing overall healthcare costs. Whole genome sequencing (WGS) has been shown to provide a rapid and comprehensive view of the genotype of the organism, and thus enable reliable prediction of the drug susceptibility phenotype within a clinically relevant timeframe. In addition, it provides the highest resolution when investigating transmission events in possible outbreak scenarios. However, robust software and database tools need to be developed for the full potential to be realized in this specialized area of medicine.
\end{abstract}

Keywords: Whole genome sequencing, Multidrug resistance, Tuberculosis

\section{Background}

Early observations made in 2008 [1] predicted the potential for whole genome sequencing (WGS) to transform tuberculosis (TB) diagnostics, when the then recent use of "next generation" sequencing technology to analyse extensively drug-resistant (XDR) isolates of Mycobacterium tuberculosis was summarized. Such has been the speed of technological advances that the description of 1 gigabase $(\mathrm{Gb})$ of sequence as "staggering" in 2008 is now dwarfed by the current state-of-the-art machines, producing up to $15 \mathrm{~Gb}$ on the benchtop MiSeq and 1,500 Gb on HiSeq 4000. The inevitable economies of scale have therefore led to a rapid drop in costs, thus opening up microbiology diagnostics to direct sequencing applications.

In order to consider the potential for WGS to improve diagnosis, treatment and management of TB in a clinical setting, it is useful to understand the current diagnostic workflow. The exact details of this process (and thus the potential impact of WGS) vary between different settings and countries, so we discuss this in the context of

\footnotetext{
* Correspondence: awitney@sgul.ac.uk

'Institute of Infection and Immunity, St George's University of London,

Cranmer Terrace, London SW17 ORE, UK

Full list of author information is available at the end of the article
}

St George's Hospital, London, UK. In 2014, a total of 6,520 cases of TB (incidence of approximately 12 per 100,000) were recorded in England, of which $1.4 \%$ were multidrugresistant TB (MDR-TB); defined as resistant to isoniazid (INH) and rifampicin (RIF) [2]. However, both TB (30 per 100,000) and MDR-TB levels are higher in London. Extensively drug-resistant TB (XDR-TB) occurs at a much lower rate, but the requirements for effective clinical management and treatment of the MDR-TB and XDR-TB and the public health management of such cases are even more onerous.

\section{Current practice}

The standard procedure for a case of TB at St George's Hospital would be as follows. A patient is referred from a primary care setting, a chest clinic or a local hospital as having suspected pulmonary TB, or attends of their own volition. A sputum sample is taken, examined by microscopy for acid-fast bacilli (AFB) and a BacT/Alert (Organon Teknika Corporation, Durham, NC, USA) [3] liquid culture inoculated. If $\mathrm{TB}$ is clinically suspected then treatment will be started immediately; otherwise, the smear results will be returned within 1-2 days, and if positive, the patient is started on standard TB treatment according to WHO guidelines $[4,5]$. 
If the M. tuberculosis is fully sensitive, this is the correct treatment regimen. However, there is a significant chance that antibiotic resistance may be present, and if true, the standard treatment may be completely ineffective, or if only partially so, could make the development of resistance more likely. Therefore, when BacT/Alert cultures become positive, usually within 1-2 weeks, they are sent to the UK's national reference laboratory (NRL) for first-line drug phenotypic susceptibility testing (DST). Results are returned within 2-3 weeks, but if evidence of resistance is found then second-line DST is begun, returning after a further $2-3$ weeks. It can therefore take up to 8 weeks after presentation before a full DST profile is available for resistant cases.

In order to speed up this process, St George's Hospital carries out molecular tests to identify likely MDR-TB cases. Following microscopy, an AFB smear-positive sputum is also tested for genetic markers of RIF resistance using the Xpert $^{\oplus}$ MTB/RIF assay (Cepheid, Sunnyvale, CA, USA) [6]; results are available within two days. Other hospitals may wait for a positive culture before performing an Xpert $^{\circledast}$ MTB/RIF assay, with the subsequent delays that may then occur. A negative Xpert ${ }^{\oplus}$ MTB/RIF assay result for RIF resistance leads to continuation of standard drugsensitive treatment, with subsequent public health action to trace contacts, assess exposure with Mantoux skin tests and offer INH prophylaxis, where appropriate. A positive $\mathrm{Xpert}^{\ominus} \mathrm{MTB} / \mathrm{RIF}$ assay result indicating RIF resistance suggests that this is now a likely case of MDR-TB, an assumption that has considerable clinical and public health consequences. It leads to an assessment of the risk factors for resistance and the clinical condition of the patient, in order to decide whether to cease first-line drug treatment and switch to a second-line drug regimen $[4,5]$ at this stage or wait for further molecular confirmation of resistance. Furthermore, heightened infection control measures are implemented and extended public health questionnaires initiated.

Other molecular tests are available, notably the GenoType MTBDRplus (Hain Lifescience, GmbH, Nehren, Germany) [7] which identifies selected markers for INH resistance in addition to RIF, further confirming MDR$\mathrm{TB}$, and the GenoTypeMTBDRsl (Hain Lifescience) [8] which also identifies selected resistance markers for fluoroquinolones, aminoglycosides and ethambutol, indicative of XDR-TB. In our context, these tests are available from the NRL but have to be specifically requested.

WGS has two main overlapping uses in clinical microbiology and public health: i) identification of genotypes which can be used to predict drug-resistant phenotype; and ii) the determination of genetic relatedness which can identify transmission chains in potential outbreak scenarios. Both of these can have direct patient benefits.

\section{Genotype-phenotype prediction}

The prediction of antibiotic resistance phenotype from genotype is not yet a solved problem; however, it is a more straightforward task for $M$. tuberculosis than for other species due to a lack of horizontal gene transfer. Indeed, drug resistance is generally thought to be mediated only through mutations in specific gene targets. The key, therefore, is to identify those single nucleotide polymorphisms (SNPs) which are responsible for, or strongly associated with, resistance.

Many studies have been performed identifying individual markers of resistance; however, more recently, several large sequencing studies have analysed clinical isolates and correlated SNPs to phenotypic drug sensitivity results, calculating predictive sensitivities and specificities of sets of SNPs [9-11]. In this context, specificity describes the likelihood that SNPs identified lead to resistance, while sensitivity describes the proportion of resistant cases that are identified through that set of SNPs. But the confidence for which a prediction can be made has been shown to vary according to not only drug class but also different members of these classes. This is slightly complicated by the fact that the gold standard (culture) has its own error rate [12].

The aforementioned molecular diagnostic tests are targeted methods for predicting the phenotype of the organism from the detected genotype. These examine a limited number of sites known to be associated with varying levels of resistance to the specific drugs mentioned. False negatives can arise, for example, when any rifampicinresistant $\left(\mathrm{RIF}^{\mathrm{R}}\right)$ mutations that lie outside the $r p o B$ RIF resistance-determining region (RRDR), such as the I491F mutation in RpoB $[13,14]$, are not detected by the Xpert ${ }^{\circ}$ MTB/RIF assay leading to the isolate being incorrectly called susceptible. Similarly, false positives can arise when a polymorphism in the RRDR region of $r p o B$ is detected and $\mathrm{RIF}^{\mathrm{R}}$ predicted when they are either completely rifampicinsensitive $\left(\mathrm{RIF}^{\mathrm{S}}\right)$ or have a low level of resistance that could be overcome by an appropriately increased dosage (see below). Furthermore, it is important to understand that resistance is not a binary phenomenon, and the variable impact of individual mutations on the minimum inhibitory concentration (MIC) raises the possibility of designing personalized treatment regimes, for example:

i) It has been established that INH resistance, predominantly mediated through loss of catalaseperoxidase activity via mutations in $k a t G$, produce high-level resistant strains (MIC: $2-8 \mu \mathrm{g} / \mathrm{ml} \mathrm{INH}$ ) and that the KatG S315T substitution occurs in 50-95\% of INH-resistant isolates [15]. However, mutations in the inhA gene or promotor region produce low-level resistant strains (MIC: $0.2-$ $0.5 \mu \mathrm{g} / \mathrm{ml}$ ) occurring in $8-43 \%$ of INH-resistant 
isolates. Therefore, if a katG high-level resistance mutation is detected, isoniazid therapy should not be included and could be substituted with prothionamide. Prothionamide has the same drug target as INH, namely InhA, but it is activated from the pro-drug form to the active drug by a different enzyme, EthA, and mutations in the eth $A$ gene might contraindicate the use of prothionamide. If, however, an $i n h A$ promotor mutation is present suggesting low-level resistance, then INH could remain in the regimen but at a higher dosage as INH has a high therapeutic margin.

ii) Moxifloxacin is a second-line drug included in standard MDR-TB regimens, and specific mutations in the gyrA gene confer resistance often suggesting the presence of an XDR-TB isolate. However, different mutations in gyrA impact MIC values differently according to the quinolone type [16]. For example, an MIC value for moxifloxacin of $1 \mu \mathrm{g} / \mathrm{mL}$ has been shown in isolates with the A90V substitution in GyrA. This would be called resistant by conventional phenotyping using the standard breakpoint of $>0.5 \mu \mathrm{g} / \mathrm{ml}$, but Feasey et al. [17] showed successful treatment by increasing the dose of moxifloxacin from 400 to $600 \mathrm{mg}$ in order to achieve in vivo drug levels above the MIC. Subsequent identification of this A90V mutation in other clinical isolates could be used as evidence to indicate similar dosage alterations for effective treatment in the face of a resistant DST result.

iii) Mutations in the 89 base pair RRDR of $r p o B$ confer a range of resistance phenotypes with large variations in MICs $[18,19]$. Knowing the exact mutation and subsequent amino acid change is therefore crucial. A case of possible MDR-TB could be indicated by a positive Xpert ${ }^{\circ} \mathrm{MTB} / \mathrm{RIF}$ assay and called $\mathrm{RIF}^{\mathrm{R}}$ because of a SNP in the RRDR that is different from wild-type, identified by one of the five probes used in that test. This SNP could be a simple phylogenetic polymorphism independent of resistance, or a SNP associated with low-level RIF ${ }^{\mathrm{R}}$. Such a false positive result would wrongly indicate MDR-TB and exclusion of RIF in the initial regimen prior to phenotypic susceptibility testing. For example, MIC data for a range of non-synonymous SNPs at codon 526 has been reported ranging from $1-256 \mu \mathrm{g} / \mathrm{ml}[18,19]$. Thus, RpoB H526N, an allele we recently found in a $M$. tuberculosis patient isolate, has an MIC of $0.25 \mu \mathrm{g} / \mathrm{ml}$, so rifampicin can still be included in the regimen despite the $\mathrm{Xpert}^{\oplus} \mathrm{MTB} / \mathrm{RIF}$ assay returning a resistant result at initial diagnosis. Furthermore, different alleles have different levels of cross-resistance to rifabutin (RFB), a semi-synthetic derivative of RIF $[19,20]$. For example, mutation
H526L is associated with a $\operatorname{RIF}^{\mathrm{R}} / \mathrm{RFB}^{\mathrm{S}}$ pattern and might inform the substitution of RFB into a therapy regimen. Sequence data might thus direct the use of either RIF or RFB depending on the exact allele identified.

A recent and comprehensive report from the TBNET and RESIST-TB networks collated genotype-phenotype data from literature and electronic databases and reached a consensus about reporting standards in the clinical use of molecular DST results [21]. The report summarised the clinical implications of mutations detected by molecular methods and provided much needed clarity on the issue of how genotypic data can be interpreted in terms of $\mathrm{TB}$ drug regimes.

\section{Determination of genetic relatedness}

In the UK, outbreak identification is currently determined using the mycobacterial interspersed repetitive unit-variable number tandem repeat (MIRU-VNTR) method [22], which can suggest clusters of isolates but lacks the resolution to be certain of possible transmission events. The identification of such events clearly has a role in public health intervention, but can also directly benefit the patient if clinical outcome data is available for the strain in question, ensuring that patients and infected contacts can be isolated and initiated on the most effective treatment as quickly as possible. We have experienced this scenario at St George's Hospital; WGS identified an XDR-TB strain from a hospital in-patient with smear-positive pulmonary $\mathrm{TB}$ and no risk factors of MDR/XDR-TB, as identical to a known XDR-TB strain that we had previously sequenced. The patient was switched to an XDR-TB regimen and subsequent sputum samples showed culture conversion.

Several studies have attempted to validate WGS for public health interventions. Gardy et al. [23] demonstrated the limited resolution of MIRU-VNTR for outbreak investigation by sequencing $32 \mathrm{M}$. tuberculosis isolates from a three year outbreak of TB in British Columbia, Canada. WGS data clearly showed the presence of two distinct lineages of M. tuberculosis with identical MIRU-VNTR types, although they had both likely descended from a historical common ancestor. Furthermore, the integration of WGS with social network information identified several transmission events and the presence of super-spreaders, leading to the conclusion that the outbreak coincided with a recorded increase in crack cocaine usage.

Török et al. [24] investigated two cases of MDR-TB associated with a language school in Cambridge, UK. The cases had an epidemiological link through the school, but there was a discrepancy in phenotypic results; one was determined streptomycin-resistant and the other sensitive. The UK's National Mycobacterial Reference Laboratory showed that both isolates were of the Beijing 
lineage and of the same MIRU-VNTR type; however, the phenotypic difference suggested that they were different strains. Subsequent WGS showed that the isolates did not display any differences in SNPs, suggesting a missed direct or indirect transmission event. This case highlights a particular problem with the reliability of phenotypic testing, and the authors suggest that MICs close to the breakpoint for streptomycin could have affected the reproducibility and thus the discrepancy between the two isolates.

Walker et al. [25] undertook a larger, retrospective observational study using WGS to delineate TB clusters within 390 isolates from 254 patients archived over a 17 -year period from the central UK region. By analysing the patterns of mutations the study enabled an inference about direction of transmission within outbreaks and also identified the existence of potential super-spreaders in several of the clusters investigated.

Pankhurst et al. [26] identified several outbreak scenarios which had been previously missed by standard routine methods, and demonstrated that WGS in routine use can identify transmission networks and direct better public health interventions.

\section{Clinical consequences}

Clinical management of antibiotic-resistant TB is complex. The drugs are often poorly tolerated, and side-effects include ototoxicity, nephrotoxicity, hepatitis, diarrhoea, vomiting and psychological disturbances [27]. Additionally, the requirement for an injectable drug in the regime requires the insertion of a central long line, which exposes the patient to an increased risk of infection and venous thrombosis. According to UK national guidelines [28], patients should be isolated in negative pressure, HEPA-filtered single occupancy rooms until 3 weekly negative smears have been obtained that are ideally also culture negative. At St George's Hospital we wait for the repeated respiratory samples to be culturenegative after a 6 week incubation. These measures are unpleasant for the patient, expensive for the hospital and compliance can be difficult. Where drug resistance is suggested, second-line MDR treatment is started in a complete absence of detailed resistance information, and DST results only appear up to 5-8 weeks after presentation. In our experience, the initial regimen is often not optimal, and when XDR-TB cases are encountered may be completely ineffective. Even when optimal, it may be desirable to change because of drug side-effects, and to do this in the absence of resistance information is not ideal.

The routine use of a genotypic RIF testing gives a faster indication of the suitability of the drug regimen. In settings where this is not done, DST for MDR-TB cases will be carried out in two phases, leading to further delays. In these cases, patients are likely to have spent the first 3-5 weeks on ineffective first-line drugs, and will only have generic MDR treatment for the subsequent 2-3 weeks.

False negative and false positive tests for resistance have significant implications for the patient. Incorrectly assigning a patient to drug-sensitive TB means many weeks of infective treatment and subsequent further resistance developing, whilst incorrectly assigning to a regime for drug-resistant TB has implications due to the drug toxicities and stringent public health measures implemented. The consequences of such errors are severe, especially when it is considered that these will only be corrected once the full DST profile is received, up to 8 weeks later. WGS avoids these specific problems by presenting an unbiased view of the whole genome; all sites are examined simultaneously, in a timeframe considerably shorter than current methods. WGS interpretation does, however, require expertise in bioinformatics and data analysis tools that may not be available in all settings.

Several studies have investigated the potential impact of WGS on patient benefit by attempting to predict drug susceptibilities from the isolate genotypes [26, 29-31]. Witney et al. [29] demonstrated that WGS can be used to provide treating physicians with valuable information in a clinically relevant timeframe, several weeks before DST results can be returned. More recently, Pankhurst et al. [26] demonstrated routine use of WGS and calculated that the system costs $£ 481$ (WGS alone), $£ 518$ (DST alone) or $£ 540$ (DST and WGS), demonstrating that WGS can be economically viable. Furthermore, these differences in costs are tiny when compared to the costs of in-patient treatment for drug-resistant TB; at St George's Hospital, we estimate that treating a TB patient in the airflowcontrolled ward costs $£ 900$ per day, not including the costs of the often expensive drugs required for MDR and XDR cases. The case for any intervention that has the potential to save weeks of patient care is therefore clear.

All the studies described so far have applied WGS to cultured isolates; however, a further 1-2 weeks could be saved if the culture step is eliminated. WGS applied direct to sputum is a difficult problem due to the low number of organisms and the presence of contaminating human and non-mycobacterial DNA. To address this problem, Brown et al. [32] used DNA sequence capture with biotinylated RNA bait oligonucleotides, spanning the entire $M$. tuberculosis genome, followed by amplification and sequencing of the captured DNA. This method was applied to 24 TB sputum specimens and showed good on-target reads and depth of coverage for 23 samples with associated accurate prediction of drug resistance mutations. Evidence for a mixed infection with two strains in one sample was also predicted from the genome sequence data and this 
indicates another potential read-out of WGS that is unlikely to be obtained through other methods. Such direct use of WGS has been proposed as the basis for personalizing therapy for drug-resistant TB [33].

WGS is not a stationary technology [34]; development of miniaturized sequencing platforms such as the MinION from Oxford Nanopore Technologies (Oxford, UK) will eventually democratize the implementation of WGS in the clinical management of TB by substantially reducing the cost, making the economic, technical and clinical arguments for WGS implementation incontrovertible [35].

\section{Bioinformatics}

Bioinformatic analysis is a key part of the WGS process, and for optimal clinical use, issues within the analysis pipelines and database resources need to be addressed.

The analysis process can be broken down into three steps: i) alignment of the DNA sequence data to a reference genome, normally the $\mathrm{H} 37 \mathrm{Rv}$ strain of $M$. tuberculosis; ii) variant site calling which determines the probability that a SNP is correctly identified in the sequence and is not due to a random error introduced during the sequencing reaction; and iii) resistance prediction or phylogenetic reconstruction. Numerous alignment tools are available and all have strengths and weaknesses [36], such that the choice is invariably made by personal preference. Variant site calling commonly involves either SAMtools [37], FreeBayes [38] or GATK [39], and resistance prediction is often implemented using custom software, comparing identified mutations with databases of known resistance-conferring SNPs. Phylogenetic reconstruction from sequence data is not a new problem, but when applied to clinical WGS data some work still needs to be done with regards to estimating the error when determining SNP number differences between isolates in potential transmission chains; the identification of a transmission event can have significant downstream clinical and public health impact.

Many software pipelines have been developed and are in use in individual laboratories or available via web interfaces $[9,39]$. However, the future implementation of WGS in clinical practice will depend on the development of robust and validated software tools that are easy to use and interpret in the diagnostic context. Promising examples are under development that can predict a phenotype within minutes of uploading sequence data, for example Mykrobe [40]. Efforts are also under way to assess and standardize methods, for example the Global Microbial Identifier group [41] initiated an open proficiency test in 2015, providing standard DNA material or sequence data for processing by participants. Although this does not currently include $M$. tuberculosis, the alignment and site calling steps described above are generally organism-independent. However, such standard reference data are essential to develop consensus, thus encouraging better inter- and intra-laboratory reproducibility.

The reliability of the genotype-phenotype prediction is dependent not only on good correlation data but also on good database resources to allow easy access to such data [42]. TBDReaMDB [43] was the first TB-specific database of drug resistance-conferring mutations, created in 2009, and more recently updated in 2014. It was collated through literature searches; however, it now accepts submissions from external users. TBDReaMDB does not attempt to validate target mutations, instead only cataloguing studies examining mutations and genes of interest. For genotyping by WGS to become effective, validated and continuously updated databases need to be developed and made available; successful examples include the public HIV Drug Resistance Database [44]. The recently funded ReSeqTB project [45], which aims to implement a single repository for validated TB drugresistant correlations, may fulfil this role; however, it is still in the early stages of development. Furthermore, it is interesting to note that the aforementioned HIV database also includes data on genotype-clinical outcome correlations. Maybe this would be a useful addition to any TB mutation repository, since sharing successful treatment options for already sequenced strains could be as effective as predicting phenotype solely from genotype. The mechanics of the interaction between the software tools and global databases of resistance mutations and phylogenetic information is yet to be determined. However, the true power of WGS will not be realised unless open and integrated systems are put in place.

Arguably WGS could be used to expand the current rapid, targeted molecular methods by improving the predictive power of any identified mutation, but whereas the targeted molecular approach requires constant technical development to maintain a comprehensive mutation detection list, WGS requires only updates to its predictive algorithm, thus improving the diagnostic power more rapidly.

\section{Conclusions}

The advantages of WGS in the TB diagnostic workflow are clear when compared to standard DST; results can be returned several weeks earlier and, in the future, this will only become faster and cheaper. Compared to current molecular tests, until WGS can be routinely performed on sputum, results will lag by 1-2 weeks. Nevertheless, the vastly increased resolution of WGS results means a more accurate and comprehensive picture of resistance prediction than is currently possible.

In addition, the ability to inform transmission detection, without the need for extra tests, and the comparability of cost ensure that the use of WGS in clinical microbiology is just a matter of how and when, rather than if. Current 
indications are that dual testing (WGS and DST) is only marginally more expensive than WGS alone, and while certainty in phenotypic resistance is not yet (if at all) possible, it would be prudent to maintain DST in the standard workflow. More research focusing on the realities of clinical management and economic benefits and constraints in different environments will be immensely valuable.

\section{Abbreviations}

AFB: acid-fast bacilli; DST: drug susceptibility testing; INH: isoniazid; MDRTB: multidrug-resistant tuberculosis; MIC: minimum inhibitory concentration MIRU-VNTR: mycobacterial interspersed repetitive unit-variable number tandem repeat; NRL: national reference laboratory; RFB: rifabutin; RIF: rifampicin; RRDR: rifampicin resistance-determining region; SNP: single nucleotide polymorphism; TB: tuberculosis; WGS: whole genome sequencing; WHO: World Health Organization; XDR-TB: extensively drug-resistant tuberculosis.

\section{Competing interests}

PB and AW are funded in part by Innovate UK (a UK government agency) in collaboration with QuantuMDx Ltd (Newcastle upon Tyne, UK). CC, AA, NS and $\mathrm{JH}$ declare that they have no competing interests.

\section{Authors' contributions}

$A W, P B, N S, A A$ and $C C$ contributed jointly to writing the manuscript. Clinical aspects of the manuscript were contributed by CC and AA. AW, PB, JH and NS implemented WGS on clinical cases at St George's University Hospitals NHS Foundation Trust, London, from which this review draws its experience. AW provided the bioinformatics insights. All authors read and approved the final manuscript.

\section{Acknowledgements}

M. tuberculosis sequencing was funded through a grant from St George's Charitable Foundation Medical Research Committee grant awarded to AA, with PB and CC. The Committee had no role in the design, collection, analysis or interpretation of the study.

\section{Author details}

'Institute of Infection and Immunity, St George's University of London, Cranmer Terrace, London SW17 ORE, UK. Clinical Infection Unit, St George's University Hospitals NHS Foundation Trust, Blackshaw Road, London SW17 OQT, UK.

Received: 13 January 2016 Accepted: 11 March 2016

Published online: 23 March 2016

\section{References}

1. Loman NJ, Pallen MJ. XDR-TB genome sequencing: a glimpse of the microbiology of the future. Future Microbiol. 2008;3(2):111-3.

2. Public Health England. Tuberculosis in the UK: 2015 report (presenting data to end of 2014). London: Public Health England; 2015. Available from: https://www.gov.uk/government/uploads/system/uploads/attachment_ data/file/464914/TB_Annual_Report_2015.pdf.

3. Thorpe TC, Wilson ML, Turner JE, DiGuiseppi JL, Willert M, Mirrett S, et al BacT/Alert: an automated colorimetric microbial detection system. J Clin Microbiol. 1990;28(7):1608-12.

4. World Health Organization (WHO). Guidelines for the programmatic management of drug-resistant tuberculosis: 2011 update. Geneva: WHO; 2011. Available from: http://www.ncbi.nlm.nih.gov/books/NBK148644.

5. Lange C, Abubakar I, Alffenaar JW, Bothamley G, Caminero JA, Carvalho ACC, et al. Management of patients with multidrug-resistant/extensively drug-resistant tuberculosis in Europe: a TBNET consensus statement. Eur Respir J. 2014;44(1):23-63.

6. Helb D, Jones M, Story E, Boehme C, Wallace E, Ho K, et al. Rapid detection of Mycobacterium tuberculosis and rifampin resistance by use of on-demand, near-patient technology. J Clin Microbiol. 2010;48(1):229-37.

7. Hillemann D, Weizenegger M, Kubica T, Richter E, Niemann S. Use of the genotype MTBDR assay for rapid detection of rifampin and isoniazid resistance in Mycobacterium tuberculosis complex isolates. J Clin Microbiol. 2005:43(8):3699-703.
8. Hillemann D, Rüsch-Gerdes S, Richter E. Feasibility of the GenoType MTBDRsl assay for fluoroquinolone, amikacin-capreomycin, and ethambutol resistance testing of Mycobacterium tuberculosis strains and clinical specimens. J Clin Microbiol. 2009:47(6):1767-72.

9. Coll F, McNerney R, Preston MD, Guerra-Assunção JA, Warry A, Hill-Cawthorne $\mathrm{G}$, et al. Rapid determination of anti-tuberculosis drug resistance from whole-genome sequences. Genome Med. 2015;7(1):51.

10. Zhang H, Li D, Zhao L, Fleming J, Lin N, Wang T, et al. Genome sequencing of 161 Mycobacterium tuberculosis isolates from China identifies genes and intergenic regions associated with drug resistance. Nat Genet. 2013;45(10):1255-60.

11. Walker TM, Kohl TA, Omar SV, Hedge J, Elias CDO, Bradley P, et al. Wholegenome sequencing for prediction of Mycobacterium tuberculosis drug susceptibility and resistance: a retrospective cohort study. Lancet Infect Dis. 2015;15(10):1193-202

12. Hillemann D, Hoffner S, Cirillo D, Drobniewski F, Richter E, Rüsch-Gerdes S, et al. First evaluation after implementation of a quality control system for the second line drug susceptibility testing of Mycobacterium tuberculosis joint efforts in low and high incidence countries. PLoS One. 2013;8(10):e76765.

13. Sanchez-Padilla E, Merker M, Beckert $P$, Jochims F, Dlamini T, Kahn P, et al. Detection of drug-resistant tuberculosis by Xpert MTB/RIF in Swaziland. N Engl J Med. 2015;372(12):1181-2.

14. Siu GKH, Zhang Y, Lau TCK, Lau RWT, Ho PL, Yew WW, et al. Mutations outside the rifampicin resistance-determining region associated with rifampicin resistance in Mycobacterium tuberculosis. J Antimicrob Chemother. 2011;66(4):730-3.

15. Jacobson KR, Theron D, Victor TC, Streicher EM, Warren RM, Murray MB. Treatment outcomes of isoniazid-resistant tuberculosis patients, Western Cape Province, South Africa. Clin Infect Dis. 2011;53(4):369-72.

16. Malik S, Willby M, Sikes D, Tsodikov OV, Posey JE. New insights into fluoroquinolone resistance in Mycobacterium tuberculosis: functional genetic analysis of gyrA and gyrB mutations. PLoS One. 2012;7(6):e39754.

17. Feasey NA, Pond M, Coleman D, Solomon AW, Cosgrove CA, Delgado R, et al. Moxifloxacin and pyrazinamide susceptibility testing in a complex case of multidrug-resistant tuberculosis. Int J Tuberc Lung Dis. 2011;15(3):417-20.

18. Pang $Y, L$ Lu J, Wang $Y$, Song $Y$, Wang $S$, Zhao $Y$. Study of the rifampin monoresistance mechanism in Mycobacterium tuberculosis. Antimicrob Agents Chemother. 2013;57(2):893-900.

19. Jamieson FB, Guthrie JL, Neemuchwala A, Lastovetska O, Melano RG, Mehaffy C. Profiling of rpoB mutations and MICs for rifampin and rifabutin in Mycobacterium tuberculosis. J Clin Microbiol. 2014;52(6):2157-62.

20. Sirgel FA, Warren RM, Böttger EC, Klopper M, Victor TC, van Helden PD. The rationale for using rifabutin in the treatment of MDR and XDR tuberculosis outbreaks. PLoS One. 2013:8(3):e59414.

21. Domínguez J, Boettger EC, Cirillo D, Cobelens F, Eisenach KD, Gagneux S, et al. Clinical implications of molecular drug resistance testing for Mycobacterium tuberculosis: a TBNET/RESIST-TB consensus statement. Int J Tuberc Lung Dis. 2016;20(1):24-42.

22. Supply P, Allix C, Lesjean S, Cardoso-Oelemann M, Rüsch-Gerdes S, Willery E, et al. Proposal for standardization of optimized mycobacterial interspersed repetitive unit-variable-number tandem repeat typing of Mycobacterium tuberculosis. J Clin Microbiol. 2006;44(12):4498-510.

23. Gardy JL, Johnston JC, Sui SJH, Cook VJ, Shah L, Brodkin E, et al. Wholegenome sequencing and social-network analysis of a tuberculosis outbreak. N Engl J Med. 2011;364(8):730-9.

24. Török ME, Reuter S, Bryant J, Köser CU, Stinchcombe SV, Nazareth B, et al. Rapid whole-genome sequencing for investigation of a suspected tuberculosis outbreak. J Clin Microbiol. 2013;51(2):611-4.

25. Walker TM, Ip CL, Harrell RH, Evans JT, Kapatai G, Dedicoat MJ, et al. Wholegenome sequencing to delineate Mycobacterium tuberculosis outbreaks: a retrospective observational study. Lancet Infect Dis. 2013;13(2):137-46.

26. Pankhurst LJ, del Ojo EC, Votintseva AA, Walker TM, Cole K, Davies J, et al. Rapid, comprehensive, and affordable mycobacterial diagnosis with wholegenome sequencing: a prospective study. Lancet Respir Med. 2016;4(1):49-58.

27. Sturdy A, Goodman A, José RJ, Loyse A, O'Donoghue M, Kon OM, et al. Multidrug-resistant tuberculosis (MDR-TB) treatment in the UK: a study of injectable use and toxicity in practice. J Antimicrob Chemother. 2011:66(8):1815-20.

28. National Institute for Health and Care Excellence (NICE). Tuberculosis. NICE guidelines NG33. London: NICE; 2016. Available from: http://www.nice.org. uk/guidance/ng33/. 
29. Witney AA, Gould KA, Arnold A, Coleman D, Delgado R, Dhillon J, et al. Clinical application of whole-genome sequencing to inform treatment for multidrug-resistant tuberculosis cases. J Clin Microbiol. 2015;53(5):1473-83.

30. Outhred AC, Jelfs P, Suliman B, Hill-Cawthorne GA, Crawford ABH, Marais BJ, et al. Added value of whole-genome sequencing for management of highly drug-resistant TB. J Antimicrob Chemother. 2015;70(4):1198-202.

31. Koser C, Bryant JM, Becq J, Torok ME, Ellington MJ, Marti-Renom MA, et al. Whole-genome sequencing for rapid susceptibility testing of $\mathrm{M}$. tuberculosis. N Engl J Med. 2013;369(3):290-2.

32. Brown AC, Bryant JM, Einer-Jensen K, Holdstock J, Houniet DT, Chan JZM, et al. Rapid Whole-genome sequencing of Mycobacterium tuberculosis isolates directly from clinical samples. J Clin Microbiol. 2015;53(7):2230-7.

33. Bryant JM, Lipman $M$, Breuer J. Personalizing therapy for multidrug resistant TB: the potential of Rapid Whole Genome Sequencing. Expert Rev Anti Infect Ther. 2016:14(1):1-3.

34. McPherson JD. A defining decade in DNA sequencing. Nat Methods 2014;11(10):1003-5.

35. Judge K, Harris SR, Reuter S, Parkhill J, Peacock SJ. Early insights into the potential of the Oxford Nanopore MinION for the detection of antimicrobial resistance genes. J Antimicrob Chemother. 2015;70(10):2775-8.

36. Hatem A, Bozdağ D, Toland AE, Çatalyürek ÜV. Benchmarking short sequence mapping tools. BMC Bioinformatics. 2013;14:184.

37. Li H, Handsaker B, Wysoker A, Fennell T, Ruan J, Homer N, et al. The Sequence Alignment/Map format and SAMtools. Bioinformatics. 2009;25(16):2078-9.

38. Garrison E, Marth G. Haplotype-based variant detection from short-read sequencing. ArXiv12073907 Q-Bio; 2012. Available from: http://arxiv.org/abs/ 1207.3907.

39. Feuerriegel S, Schleusener V, Beckert P, Kohl TA, Miotto P, Cirillo DM, et al. PhyResSE: a web tool delineating Mycobacterium tuberculosis antibiotic resistance and lineage from whole-genome sequencing data. J Clin Microbiol. 2015;53(6):1908-14.

40. Bradley P, Gordon NC, Walker TM, Dunn L, Heys S, Huang B, et al. Rapid antibiotic-resistance predictions from genome sequence data for Staphylococcus aureus and Mycobacterium tuberculosis. Nat Commun. 2015;6:10063.

41. Global Microbial Identifier (GMI). Available from: http://www. globalmicrobialidentifier.org/.

42. Stucki D, Gagneux S. Single nucleotide polymorphisms in Mycobacterium tuberculosis and the need for a curated database. Tuberculosis (Edinb). 2013:93(1):30-9.

43. Sandgren A, Strong M, Muthukrishnan P, Weiner BK, Church GM, Murray MB Tuberculosis drug resistance mutation database. PLoS Med. 2009;6(2):e2

44. Shafer RW. Rationale and uses of a public HIV drug-resistance database. J Infect Dis. 2006;194 Suppl 1:S51-8.

45. Relational Sequencing TB Data Platform (ReSeqTB). Available from: https://platform.reseqtb.org/.

\section{Submit your next manuscript to BioMed Central and we will help you at every step:}

- We accept pre-submission inquiries

- Our selector tool helps you to find the most relevant journal

- We provide round the clock customer support

- Convenient online submission

- Thorough peer review

- Inclusion in PubMed and all major indexing services

- Maximum visibility for your research

Submit your manuscript at www.biomedcentral.com/submit

) Biomed Central 